\title{
Patient adherence to multivitamin supplementation after bariatric surgery: a narrative review
}

\author{
Hendrika J.M. Smelt ${ }^{1,2} *$, Sjaak Pouwels ${ }^{3}$, Johannes F. Smulders ${ }^{1,2}$ and Eric J. Hazebroek ${ }^{4,5}$ \\ ${ }^{1}$ Department of Surgery, Catharina Hospital, Michelangelolaan 2, P.O. Box 1350, Eindhoven 5602 ZA, The Netherlands \\ ${ }^{2}$ Obesity Center, Catharina Hospital, Eindhoven, The Netherlands \\ ${ }^{3}$ Department of Intensive Care Medicine, Elisabeth-Tweesteden Hospital, Tilburg, The Netherlands \\ ${ }^{4}$ Department of Surgery, Rijnstate Hospital, Arnhem, The Netherlands \\ ${ }^{5}$ Division of Human Nutrition and Health, Wageningen University and Research, Wageningen, The Netherlands
}

(Received 25 August 2020 - Final revision received 4 September 2020 - Accepted 7 September 2020)

Journal of Nutritional Science (2020), vol. 9, e46, page 1 of 8

doi:10.1017/jns.2020.41

\section{Abstract}

Morbid obesity is a growing problem worldwide and has subsequently resulted in a wide application of bariatric surgery to achieve long-term weight loss and improvement of obesity-related co-morbidities. In spite of these clinical benefits, vitamin deficiencies are common after bariatric surgery; therefore, lifelong multivitamin supplementation (MVS) is recommended. However, patient adherence to MVS intake is generally poor. The aim of this narrative review is to analyse which factors influence the adherence of MVS intake after bariatric surgery. To provide an extensive overview, we will discuss the different factors that influence MVS use in patients who underwent bariatric surgery, but also review the literature on MVS in other patient groups.

Key words: Bariatric surgery: Metabolic surgery: Patient compliance: Patient adherence: Multivitamin supplementation: Multivitamin intake

\section{Introduction}

Morbid obesity is a growing problem worldwide, which has led to a significant parallel growth in bariatric surgical procedures. Laparoscopic sleeve gastrectomy (LSG) and laparoscopic Roux-en-Y gastric bypass (LRYGB) are currently the most common performed procedures worldwide, although more recently the One Anastomosis/Mini-Gastric Bypass (OAGB/ MGB) has gained popularity ${ }^{(1)}$. Bariatric surgery results in rapid weight loss and reduction of obesity-related co-morbidities such as hypertension, hypercholesterolaemia, diabetes mellitus type 2 and obstructive sleep apnoea syndrome $^{(2)}$. In spite of multiple clinical benefits, bariatric surgery can lead to deficiencies in macronutrients and micronutrients as a consequence of reduced intake, changes in eating pattern, food intolerance, gastrointestinal symptoms and malabsorption $^{(3,4)}$. This is confirmed by the recently published systematic review by Zarshenas et al. who found an unbalanced nutritional diet with inadequate protein intake and micronutrients in many included studies ${ }^{(5)}$.

Multivitamin supplements (MVSs) are routinely recommended lifelong to prevent vitamin deficiencies ${ }^{(4,6-10)}$. However, vitamin deficiencies are quite common after bariatric surgery despite the use of MVS, which can lead to serious long-term complications ${ }^{(3,11-13)}$. Zarshenas et al. reported about an inconsistent adherence of MVS intake after bariatric surgery $^{(5)}$. Many other studies have shown that long-term adherence of bariatric patients to MVS intake is poor ${ }^{(10,12-18)}$. However, it is unclear which factors contribute to patient

Abbreviations: LRYGB: laparoscopic Roux-en-Y gastric bypass; LSG: Laparoscopic sleeve gastrectomy; MVS: multivitamin supplementation; OAGB/MGB: One Anastomosis/Mini-Gastric Bypass

* Corresponding author: Hendrika J.M. Smelt, email marieke.smelt@catharinaziekenhuis.nl

(C) The Author(s), 2020. Published by Cambridge University Press on behalf of The Nutrition Society. This is an Open Access article, distributed under the terms of the Creative Commons Attribution-NonCommercial-ShareAlike licence (http://creativecommons.org/licenses/by-nc-sa/4.0/), which permits non-commercial re-use, distribution, and reproduction in any medium, provided the same Creative Commons licence is included and the original work is properly cited. The written permission of Cambridge University Press must be obtained for commercial re-use. 
adherence in taking MVS. Zarshenas et al. also described that further longer term and more robust studies are needed to assist healthcare professionals in providing nutritional care for bariatric surgery patients ${ }^{(5)}$.

The aim of this narrative review is to analyse which factors have an influence on the adherence of MVS intake after bariatric surgery, which could be complementary to the study by Zharshenas et al. Insights in determinants of behaviour are therefore important if healthcare professionals want to optimise therapeutic adherence ${ }^{(19)}$. To give an extensive overview, we will discuss the different factors that influence MVS use in patients who underwent bariatric surgery, but also review the literature on MVS in other patient groups.

\section{Methods}

PubMed and The Cochrane Library were searched from the earliest date of each database up to May 2020. The following keywords were used: bariatric surgery, metabolic surgery, multivitamin supplementation (MVS), multivitamin intake, patient compliance and patient adherence.

The literature on the adherence of MVS intake after bariatric surgery is very limited. As a result, the search results were too limited to perform a systematic review. Therefore, a narrative review was chosen in which all the available literature was included. Afterwards, all references of all publications were checked to not miss important publications.

The following subheadings were used in this narrative review: (1) patient-related factors, (2) therapy-related factors, (3) psychosocial and economic factors, and (4) healthcarerelated factors. This classification was established by our research group based on the studies by Jin et al. and Osterberg and Blaschke ${ }^{(19,20)}$.

The terms 'adherence' and 'compliance' are widely used in the literature. In the world of bariatric surgery, the term adherence is most commonly used in the world of bariatric surgery. The term 'adherence' is therefore used in this narrative review to aim for clarity.

Our research group decided to analyse other patient groups as well, because the available literature on patient adherence after the bariatric surgery was too limited.

\section{Patient after the bariatric surgery}

Patient adherence to MVS intake is a complex problem, which is largely unsolved in current bariatric practice. In general, adherence for MVS intake is considered to be poor in the long term. A prospective analysis by Ben-Porat et al. described the prevalence of deficiencies and supplement consumption 4 years after $\mathrm{LSG}^{(16)}$. A significant decrease in adherence over the postoperative course was documented for MVS intake (92.6 v. $37 \%$ for 1 and 4 years, $P<0.001$ ), vitamin $\mathrm{D}$ intake $(71.4 v .11 \cdot 1 \%$ for 1 and 4 years, $P<0.001)$ and calcium $(40.7$ v. $3.7 \%$ for 1 and 4 years, $P 0.002)^{(16)}$. Ledoux et al. described long-term nutritional deficiencies based on adherence to a standardised MVS after LRYGB ${ }^{(10)}$. Non-adherence patients had more deficiencies than adherence patients $(4.2 \pm 1.9 \mathrm{v}$. $2 \cdot 9 \pm 2 \cdot 0$ per deficiency per patient, $P<0 \cdot 01$ ), and the number of patients with more than five deficiencies was significantly higher in the non-compliant patient group $(P<0 \cdot 05)^{(10)}$. Non-adherence patients developed more vitamin deficiencies than good adherence patients ${ }^{(10,16)}$.

Bariatric surgery-related factors. Postoperative complaints can cause nausea, bloating, gastroesophageal reflux disease or dysphagia, which can lead to an inadequate food or MVS intake ${ }^{(21)}$. One of the most common complaints is vomiting, occurring in $30 \%$ of patients in the first postoperative period after $\operatorname{LSG}^{(4,21)}$. Several other causes have been described in the literature: food intolerance, stomal outlet stenosis/obstruction, marginal ulceration, intestinal obstruction, symptomatic gallstones, medication and dumping syndrome ${ }^{(21)}$. Prolonged vomiting can result in nutritional deficiencies ${ }^{(4)}$. Also, diarrhoea can occur due to early or late dumping syndrome, malabsorption, lactose or fructose or other food intolerances or bacterial overgrowth $^{(4,21,22)}$. Disturbed eating behaviour like inadequate chewing, over distention of the pouch by fluids, large volume meals, unhealthy product choice and simultaneous eating and drinking are major factors in developing these complaints ${ }^{(3,21)}$. This implies that counselling bariatric patients prior to surgery to modify their eating behaviour should be recommended.

Patient-related factors. Age and sex could be contributing factors for MVS adherence following the bariatric surgery, but this impact is controversial. Particularly in adolescent bariatric patients, adherence with MVS intake appears to be low ${ }^{(4)}$. One of the possible explanations is that if adolescents initially experience problems with MVS intake, they never re-initiate this behaviour which could lead to a decline in adherence over time ${ }^{(3)}$. Modi et al. assessed multivitamin adherence in forty-one adolescents after the bariatric surgery in a prospective observational study ${ }^{(3)}$. In their study, no significant differences were found between baseline age and patient adherence. A prospective study by Ben-Porat et al. assessed the prevalence of vitamin deficiencies and supplement consumption 4 years after LSG, which show no significant differences between MVS intake and age or $\operatorname{sex}^{(16)}$.

A prospective cross-sectional study by Sunil et al. analysed the relationship between vitamin adherence and demographic or psychological factors after the bariatric surgery ${ }^{(18)}$. Non-adherence was associated with male sex and employment (full-time work).

Therapy-related factors. The MVS regimen could have a major impact on patient adherence, because taking several pills every day is a problem for many bariatric patients ${ }^{(4)}$. Forgetting MVS and difficulty swallowing MVS are the two primary barriers identified for all assessment points by Modi et al. (all studied assessment points were forgetting, inconvenience, too expensive, difficult to understand doctors instruction, hard to swallow, dosing does not match my 
lifestyle, side effects and would rather do something else) ${ }^{(3)}$ (see also 'patient-related factors').

The composition of the MVS also has a major influence on the effect. Disintegration properties of the MVS are critical factors after the bariatric surgery ${ }^{(3,22)}$. The solubility and surface area are compromised by malabsorptive procedures, which influence drug absorption and bioavailability. Reduction of functional gastrointestinal capacity after the bariatric surgery could lead to reduced MVS bioavailability. MVS with a long absorptive phase will have compromised dissolution and absorption. Therefore, slow-release MVS should be avoided after the bariatric surgery. In addition, the solubility of MVS is affected by $\mathrm{pH}$ due to the decreased production of hydrochloric acid ${ }^{(22,23)}$. Literature on the disintegration properties of MVS in bariatric surgery patients is limited and should be the subject of future research ${ }^{(24)}$.

Psychosocial and economic factors. It is generally accepted that psychopathological conditions and emotional support from friends and family may have an impact on the clinical outcome. However, no well-designed studies have studied this impact on MVS adherence in bariatric surgery patients.

The costs of treatment with MVS have always been considered a major barrier to adequate lifelong adherence ${ }^{(4,25,26)}$. Patients believe that the costs of specialised MVS do not weigh up to the benefits which can lead to lower adherence ${ }^{(26)}$. Homan et al. assessed the cost-effectiveness of high-dose specialised multivitamins (WLS Forte; weight loss surgery) and over-the-counter (regular) $\mathrm{MVS}^{(26)}$. In terms of costs, there is a price difference between specialised WLS Forte and regular MVS: $€ 30 v$ v. €21, respectively. However, patients in the regular MVS group developed significantly more vitamin deficiencies $(30 \%)$ compared with the WLS Forte group $(14 \%)$. Therefore, the costs for the healthcare system are significantly higher for patients that use regular MVS in case of more vitamin deficiencies due to additional return visits and associated costs for medical staff. Total costs per patient for preventing and treating nutritional deficiencies were $€ 306$ for regular MVS and $€ 216$ for WLS Forte every 3 months. In terms of incremental costs per patient, the WLS Forte was less $\operatorname{costly}^{(26)}$.

Healthcare related factors. Lier et al. have performed a randomised controlled trial (RCT) in patients eligible for bariatric surgery, where a preoperative counselling group and a control group without preoperative counselling were compared on patient adherence to treatment guidelines ${ }^{(27)}$. Preoperative counselling consisted of improvement of coping skills to initiate and maintain postoperative lifestyle changes. Results showed no significant differences in recommended daily MVS intake (87 v. $86 \%$ for the intervention group and the control group, respectively, $P$ 0.981). Preoperative counselling did not increase MVS adherence ${ }^{(27)}$.

Ledoux et al. performed a long-term prospective study of nutritional deficits based on adherence to a standardised nutritional care after $\mathrm{LRYGB}^{(10)}$. Non-adherence patients had more vitamin deficiencies than adherence patients $(4 \cdot 2 \pm 1 \cdot 9$ v. $2.9 \pm 2.0$ deficiencies per patient, $P<0.01$ ), and the number of vitamin deficiencies correlated with the time from the last visit $(r 0.285, P<0 \cdot 01)$. Time from the last visit was significantly higher in non-adherence patients with a gap of 22 months $(11.9 \pm 1.5$ v. $34.1 \pm 8.3$ months for adherence and non-adherence patients, respectively) ${ }^{(10)}$.

There are no data how knowledgeable healthcare professionals are at recognising and prescribing appropriate dosage formulations after the bariatric surgery ${ }^{(2)}$. The literature was searched for the influence of postoperative bariatric visits and postoperative psychological and behavioural medicine visits, but these subjects were not investigated in the bariatric patient population.

What can we learn from topics of adherence in patients with other chronic diseases?

\section{Patients with other chronic diseases}

Patient-related factors. Jin et al. performed a systematic review of 102 included articles on patient adherence in general $^{(19)}$. Studies with a very specific patient population were eliminated to make this review generalisable to the general patient population. In this study, age was correlated to patient adherence ${ }^{(19)}$. This effect of age could be divided into three groups: the young group $(<40$ years $)$, the middle-age group (40-54 years) and the elderly group ( $>55$ years). Patient adherence in the middle-age group increased with increasing age. Overall, a higher adherence was seen in the elderly group ${ }^{(19)}$. However, no correlation was found between adherence and age in the cross-sectional questionnaire study by Yavuz et al., which studied the influence of patient characteristics and behaviour loss on

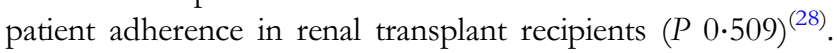
Contradictory, the adherence among men was lower than among women (P 0.087). Patients who smoke and/or drink alcohol during the pre- and post-transplant periods are more often non-adherence $(P \quad 0.008$ and 0.03 for smoking and alcohol, respectively) ${ }^{(28)}$.

A cross-sectional survey by Stone et al. examined the relationship between antiretroviral medication regimen complexity and patient understanding of the correct regimen dosing to adherence in woman with HIV/AIDS ${ }^{(29)}$. No association was found between adherence and race or ethnicity ${ }^{(29)}$, which is confirmed by the review of Osterberg and Blaschke ${ }^{(20)}$. Kaplan et al. describes the opposite in a study about sociocultural characteristics that predict non-adherence with lipid-lowering medication by patients' self-assessment of medication taking practice ${ }^{(30)}$. Independent predictors of nonadherence in multivariate analysis were race (OR 3.7, $P<$ $0 \cdot 01)$, unmarried status (OR 2.1, $P<0 \cdot 01)$ and lack of insurance (OR 2.4, $P$ 0.05) ${ }^{(30)}$. The influence of education level on adherence can be considered contradictory as well: no associations were found by Stone et al. ${ }^{(29)}$, while an association was found between good employment and adherence $\left(\begin{array}{l}P \\ 0.01)\end{array}\right.$ the prospective telephone survey by Shaw et al. to analyse factors associated with non-adherence in 243 hypertensive patients who used antihypertensive medication ${ }^{(31)}$. In addition, patient adherence tended to increase with educational 
background (P 0.059) by Yavuz et al. ${ }^{(28)}$. However, the review by Jin $e t$ al. suggests that patients with lower educational levels may put more trust in the advice of healthcare professionals ${ }^{(19)}$. Patients with a low income are more likely to be non-adherence ${ }^{(30,32)}$, whereas costs of medical therapy pose less of a problem if patients have a higher income ${ }^{(19)}$. At the same time, adherence may be threatened if patients are not able to take time off from work for healthcare treatment ${ }^{(31,33)}$.

Therapy-related factors. The complexity of treatment regimen is a major predictor of poor adherence, and this is inversely proportional to dosage frequency ${ }^{(20)}$. Long duration of the medical treatment period may adversely affect adherence as well ${ }^{(34)}$. Some studies are elaborated for illustrative purposes. Farmer et al. used prescription claim records of calcium channel blocking agents ( $n$ 9807) to determine the mean adherence ratio over a period of 2 years $^{(34)}$. The mean adherence ratio was $78.2 \%$, and the associated factors were the number of daily doses $(P<$ $0.001)$ and the length of treatment regimen $(P<0 \cdot 001)$. Once-daily regimen provides the highest adherence of 84.9 $\%$ followed by twice-daily regimen $(79.9 \%)$, three times daily regimen $(75.2 \%)$ and four times daily regimen $(73.1$ $\%)$. Once- and twice-daily regimens differ significantly $(P<$ $0 \cdot 05)^{(34)}$. Claxton et al. performed a systematic review of the association between dose regimens and medication adherence, and seventy-six studies were included ${ }^{(35)}$. Mean dose-taking adherence was $75 \%$ (range 34-97\%), and patient adherence decreased as the number of daily doses increased: $79 \pm 14 \%$ for once-daily regimen followed by twice-daily regimen $69 \pm 15 \%$, three doses daily $65 \pm 16 \%$ and four doses daily $51 \pm 20 \% \quad(P<0 \cdot 001)$. Significant differences in adherence were seen between one $v$. three doses daily $(P \quad 0.008)$, one $v$. four doses daily $(P<0.001)$ and two $v$. four doses daily ( $P$ 0.001). One $v$. two doses daily and two $v$. three doses daily show no significant difference ${ }^{(35)}$. Iskedjian et al. reported a high adherence rate for once-daily antihypertensive medication regimen $(91.5 \pm$ $2.2 \%)$ compared with a twice-daily regimen $(90 \cdot 8 \pm 4.7 \%$, $P 0.026)$ and multiple-daily dosing regimen $(83.2 \pm 3.5 \%, P$ $<0 \cdot 001)^{(36)}$. Therapeutic non-adherence is associated with poor treatment outcomes ${ }^{(37)}$. For example, poor therapy adherence results in poorly controlled blood pressure which increases the risk of myocardial ischaemia, stroke or renal impairment ${ }^{(19)}$. Paes et al. evaluated the impact of dose frequency on adherence in patients who used oral antidiabetic agents ${ }^{(38)}$. Patients received these antidiabetic drugs in a medication event monitoring system container. Each opening of the package was registered, and a questionnaire was completed at the time of the study ( $n$ 91). Overall adherence was $74.8 \%$ with an average of $79 \%$ in one-daily doses regimen and $38 \%$ in three doses daily $(P<$ $0 \cdot 01$ ). Overconsumption was occur, because one-third of this patients used more doses than prescribed ${ }^{(38)}$.

Hungin $e t$ al. determined factors associated with adherence using diary cards and questionnaires in patients with chronic use of the proton pump inhibitor (PPI) $\left(\begin{array}{ll}n & 158\end{array}\right)^{(39)}$.
Questionnaires showed an adherence rate of $70.9 \%$ taking the PPI on a once-daily regimen followed by $15.8 \%$ on most days and $13.3 \%$ took them sometimes ${ }^{(39)}$. Diaries showed complete adherence in nine patients and other patients take their medicines on less than $50 \%$ of the days.

Overall, predominant barriers of non-adherence were the length of treatment period ${ }^{(34)}$, daily dose frequency ${ }^{(34-36)}$, dose omission ${ }^{(36,38)}$, personal preference about when to take the medicine ${ }^{(39)}$, fear of side effects ${ }^{(39)}$ and medication knowledge ${ }^{(40,41)}$. Adverse effects of medical therapy have a major influence as these effects may cause physical discomfort and scepticism about the efficacy of the prescribed medication and subsequently a lowered trust in healthcare professionals $^{(32,42)}$.

Psychosocial and economic factors. Patients' beliefs about causes and meaning of illness and motivation are strongly associated with their adherence of medical therapy ${ }^{(43)}$. Adherence is better if patients feel susceptible to the illness, believe that illness or its complications pose severe consequences for patients' health and believe that the medical therapy will be effective and beneficial ${ }^{(40,44)}$. Contrarily, erroneous beliefs or misconceptions may contribute to poor adherence, and fear or negative attitude towards medical therapy is a strong predictor of poor adherence ${ }^{(19,41)}$. Gascon et al. identified factors associated with non-adherence in patients with hypertension using antihypertensive medicines ${ }^{(41)}$. A qualitative study with seven focus groups was performed. Patients' beliefs and attitude towards antihypertensive drugs and about hypertension were identified as influencing treatment adherence: fears about the long-term use of medication ('long-term use of antihypertensives is damaging'), being stuck with antihypertensive medication for life, negative feelings about the medication ('antihypertensives are damaging') and adverse effects. It was also noted that patients self-experimented with the antihypertensive doses when their blood pressure was controlled ('disease is cured when my blood pressure is controlled'). In addition, many patients stop their medication to see how they feel without it, due to low awareness about treatment, risk factors and the complications of hypertension ${ }^{(41)}$.

Sewitch et al. prospectively identified factors of nonadherence to medication in outpatients with established inflammatory bowel disease $(n 153)^{(45)}$. Non-adherence was predicted by disease activity (OR $0.55, P 0.002)$, disease duration $(P<0.001)$, scheduling a follow-up appointment $(P<$ $0 \cdot 001)$ and certainty that medication would be helpful $(P$ $0 \cdot 040)^{(45)}$.

Forgetfulness $(30 \%)$ was another major factor resulting in poor adherence ${ }^{(20)}$.

Emotional support reduces negative behaviour and attitude to the therapy and improves motivation and remembering to implement the therapy ${ }^{(19,43)}$. The influence of emotional support on adherence of adolescents with chronic disease (asthma, epilepsy, juvenile rheumatoid arthritis and diabetes mellitus) was studied by Kyngas and Rissanen in a prospective 
questionnaire study $\left(n\right.$ 1061) ${ }^{(43)}$. Logistic regression was used to indicate the good adherence predictors. Support from healthcare professionals, friends and family are statistically significant factors in predicting adherence. Support from nurses was the most powerful predictor (OR 7.28; $95 \%$ CI 3.9513.42, $P<0.001$ ) followed by support from physicians (OR 3.42; $95 \%$ CI 1.87-6.25, $P<0 \cdot 001$ ), parents (OR 2.69; 95 $\%$ CI 1.42-5.08, P 0.002) and friends (OR 2.11; $95 \%$ CI $1 \cdot 28-3 \cdot 48, P 0 \cdot 004)$, all compared to patients without support. Other interesting powerful predictors were energy and willpower to take care of themselves complied with treatment regimens (OR 6.69; $95 \% \mathrm{CI} 3.91-11.46, P<0.001)$ and motivation (OR 5.28; $95 \%$ CI 3.02-9.22, $P<0.001$ ), compared with patients without energy, willpower and motivation $^{(43)}$.

Therapeutic non-adherence leads to an increased financial burden for society, because it is associated with more emergency care visits, hospitalisations and higher treatment costs ${ }^{(19,46)}$. Of all medication-related hospital admissions in Australia and in the USA, respectively, 25 and 33-69\% are due to poor medical therapy adherence ${ }^{(19,20)}$. Svarstad et al. used drug claims data of mentally ill patients to assess the association of medication adherence (neuroleptic, lithium and antidepressant) with hospitalisation and costs ${ }^{(46)}$. Irregularly medication use was observed in $31 \%$ of patients with schizophrenia or schizoaffective disorder, $33 \%$ in patients with bipolar disorder and $41 \%$ in patients with other severe mental illness. Irregular medication users had significant higher rates of hospitalisation in all groups compared with regular users: more hospital days $(16 v 4$ days, $P<0 \cdot 01)$ and higher hospital costs $(\$ 3992 v \cdot \$ 1048, P<0 \cdot 01)^{(46)}$. In addition, psychological problems such as depression, anxiety, anger or fears about the illness are major predictors for patient adherence ${ }^{(19,30)}$.

Furthermore, medical therapy costs or co-payment were found to be associated with non-adherence as the treatment period could be lifelong ${ }^{(19,31,47)}$. Ellis et al. analysed the influence of medicine costs on adherence in patients using statin for primary and secondary prevention $\left(\begin{array}{ll}n & 4802\end{array}\right)^{(47)}$. Increasing medicine treatment costs had a large negative effect on adherence: $76 \cdot 2 \%$ non-adherence with costs of $\$ 20 /$ month $v .49 .4 \%$ non-adherence with costs of less than $\$ 10$ / month. Patients who payed $\$ 10$ till $\$ 20 /$ month were 1.45 times more likely to be non-adherence, compared with medicine costs less than $\$ 10 /$ month (OR 1.45; $95 \%$ CI 1.25 1.69). Patients who paid more than $\$ 20 /$ month were 3.23 times more likely to be non-adherence, compared with medicine costs less than $\$ 10 /$ month (OR 3.23; $95 \%$ CI 2.55-4.10) ${ }^{(47)}$.

Healthcare related factors. There are many methods available for measuring adherence, but no method is considered the gold standard. However, patient questionnaires and selfreports are described as simple, inexpensive and most useful methods in a clinical setting ${ }^{(20)}$.

Patient's satisfaction with clinical visits improved their medical therapy adherence ${ }^{(41,42)}$. However, the lack of accessibility and availability to healthcare and long waiting time for clinic visits contributed to poor adherence $e^{(19,33,41,48)}$. Major predictors associated with poor adherence are an inadequate follow-up or discharge planning, poor provider-patient relationship and missed appointments ${ }^{(44,45,47,48)}$. Spikmans et al. analysed the reasons for non-adherence for nutritional care clinics in patients with diabetes mellitus in a cross-sectional survey study ${ }^{(48)}$. One-third of these patients skipped one or more dietician visits. Non-adherence in the clinic was associated with satisfaction with the dietitian, risk perception and feelings of obligation to attend ${ }^{(48)}$.

Sewitch et al. reported total patient-physician discordance as a predicted factor of non-adherence $(P 0 \cdot 01)^{(45)}$. Gascon et al. described major predictors in the patient-doctor interaction: patient-doctor interaction not encouraged, short time consultation, little time is spent regarding information, difficulty to understand doctor's language, eye contact is rarely made during consultation and clinical encounter created nervousness. In addition, information is provided mostly upon request by the patient and just a few questions asked by the doctor ('there is not really any conversation, the doctor is explaining what's wrong and he doesn't even look at you'), and information is too general and not tailored to patients individual ('the doctor gives you advice, but he don't tell how to practice it' ${ }^{(41)}$. The overall ability of healthcare professionals to recognise patient non-adherence is poor ${ }^{(20)}$.

Using a mobile phone reminder app probably could improve patient adherence of medical therapy. The effect of mobile phone text messaging for medication adherence in patients with chronic disease was described in the meta-analysis by Thakkar et al. ${ }^{(49)}$. Sixteen RCTs with a total of 2742 patients were included (five of personalisation, eight using two-way communication and eight using a daily text message frequency). Text messaging significantly improved medication adherence from 50 to $67.8 \%$, which is promising. The authors advise to interpret the results carefully, due to the short follow-up and reliance on self-reported medication adherence measurements. Ramsey et al. published a pilot investigation of a mobile phone application and progressive reminder system to improve medication adherence in thirty-five patients with migraine ${ }^{(50)}$. Medication adherence was significantly improved in older patients with a lower baseline adherence during the first month of this study. Self-reported app-based adherence rates were significant lower when compared with electronically monitored adherence rates.

Future research needs to examine the effect of features of mobile phone message or reminder apps, appropriate patient populations, the influence on clinical outcomes and sustained long-term effects ${ }^{(49,50)}$.

Table 1 gives an overview of the described barriers that influence patient adherence in bariatric surgery patients and other patient populations.

\section{Discussion}

The long-term adherence of MVS intake after the bariatric surgery is often poor and underlying factors are unclear. This narrative review analysed which factors have an influence on the adherence of MVS intake after the bariatric surgery. Although 
Table 1. Factors that influence patient adherence of medical therapy in bariatric patients and other patient populations.

\begin{tabular}{|c|c|c|}
\hline & Bariatric patients & Other patient groups \\
\hline Patient-related factors & $\begin{array}{l}\text { Age Sex Employment Postoperative } \\
\text { complications Postoperative complaints } \\
\text { Eating behaviour }\end{array}$ & Age Education levels Employment and income \\
\hline Therapy-related factors & $\begin{array}{l}\text { Forgetting Swallowing Disintegration properties } \\
\text { of MVS }\end{array}$ & $\begin{array}{l}\text { Duration of the medical treatment Frequency of dose Convenient way of } \\
\text { administration Adverse effects of medication }\end{array}$ \\
\hline $\begin{array}{l}\text { Psychosocial and } \\
\text { economic factors }\end{array}$ & Costs of MVS treatment & $\begin{array}{l}\text { Patients lack belief in the benefit of treatment Erroneous beliefs or } \\
\text { misconception Negative attitude towards medication Treatment of } \\
\text { asymptomatic disease Presence of psychological problems } \\
\text { Forgetfulness or other priorities Emotional support Costs of medical } \\
\text { therapy }\end{array}$ \\
\hline Healthcare-related factors & Annually medical visits & $\begin{array}{l}\text { Satisfaction with clinical visits Inadequate follow-up due to missing } \\
\text { appointments Discharge planning Poor patient-doctor relationship } \\
\text { Mobile phone message or reminder apps }\end{array}$ \\
\hline
\end{tabular}

MVS, multivitamin supplement.

data on the influence of demographic characteristics are limited and contradictory, many potential causes for poor MVS adherence in bariatric patients have been identified ${ }^{(3,4,16,18)}$. Among these the most important are eating behaviour ${ }^{(3,21)}$, postoperative complications leading to gastrointestinal symptoms ${ }^{(4,21,22)}$, treatment complexity (daily pill frequency) ${ }^{(4)}$, composition of $\operatorname{MVS}^{(3,22,23)}$ and costs of MVS treatment ${ }^{(4,25,26)}$. Another important topic is that patients often believe that the costs of specialised MVS do not weight up to the benefits, which can lead to lower adherence ${ }^{(26)}$. However, the available literature on the influence of these topics in bariatric surgery patients is limited. Knowledge gained from studies in other patient populations may therefore be useful for increasing long-term adherence. Major therapy-related factors are described more extensive in other patient populations. The complexity of treatment is a major predictor of poor adherence, and this is inversely proportional to dosage frequency and have been studied in many different patient populations, as well as the duration of medication treatment, side effects and medication knowledge ${ }^{(20,34-36,38-41)}$. The absence of disease symptoms worsened patient adherence $^{(41)}$. Patients lack of belief in the benefit of treatment, have erroneous beliefs or experience misconception. Therefore, negative attitudes towards medication may have negative effects on patient adherence ${ }^{(41,43)}$. The absence of emotional support, low satisfaction with clinical visits, inadequate follow-up due to missing appointments, discharge planning and a poor patient-doctor relationship are studied in many different patient groups and are associated with poor adherence $^{(19,20,33,41-45,47,48)}$. Perhaps, the most challenging objective for healthcare professionals is to have their patients compliant to the lifelong use of medical therapy. Early recognition and intervention may improve patient adherence. Overall, the ability of healthcare professionals to recognise patient non-adherence is poor ${ }^{(20)}$. They contribute to poor adherence by failing to explain the benefits and side effects, by prescribing complex medical therapy regimens, not giving consideration to a patient's lifestyle or the costs of the treatment and having a poor therapeutic relationship with their patients as the most important factor ${ }^{(20)}$. Not knowing patients' priorities may have a high potential for low adherence $^{(41)}$. However, the doctor-patient interaction on MVS adherence in bariatric patients remains poorly understood. Our hypothesis is that patients want to please the doctor due to the discrepancy between what the patient tells and what the patient actually does. This emphasises the importance of a good doctor-patient relationship.

When a patient's condition or illness is not responding to MVS, poor adherence should always be considered. One of the factors that leads to lower adherence is the belief patients have that the costs of specialised MVS do not weigh up to its benefits. Therefore, healthcare professionals should pay attention to explain the benefits and side effects when prescribing complex MVS regimens, hereby given consideration to a patient's lifestyle and the costs of treatment. Patients' perceptions and their personal and social circumstances are crucial to their decision-making. An irrational act of non-adherence from the doctor's point of view may be a very rational action from the patient's point of view. Thus, the solution lies not in attempting to increase patient adherence, but in the development of a more open, co-operative doctor-patient relationship ${ }^{(51)}$. Enhancing communication between healthcare professionals and patients is an effective strategy boosting the patient's ability to follow a medication therapy regimen ${ }^{(20)}$. Other important issues are the daily dose regimen in bariatric patients and the prescription of supplements in the absence of symptoms. Bariatric patients often use three or four vitamin tablets daily, while literature in other patient populations shows that a simple regimen with one pill once a day helps to maximise adherence ${ }^{(20,36)}$. Gastrointestinal symptoms or incorrect eating techniques probably play a very important role in taking MVS after the bariatric surgery, while patients often think that these symptoms are caused by the MVS adherence. However, this remains the subject of further studies. Another contributing factor is a significant postoperative change in taste following bariatric surgery such as a decrease in the intensity of taste, aversion to certain food types ${ }^{(52)}$. But the most important factor is a proper formulation of the supplements, which requires consideration of the biological, physical and chemical characteristics of all of the drug substances and pharmaceutical ingredients to be used in fabricating the product ${ }^{(53)}$. Pharmaceutical and drug 
materials utilised must be compatible. Successful development of a formulation includes multiple considerations involving the drug, storage, packaging, stability and excipients. The proper combination of taste, appearance, flavour and colour in a pharmaceutical product contributes to its acceptance and a better adherence ${ }^{(53)}$. However, these important pharmaceutical points are not studied in the bariatric patient population.

Limitations of this narrative review are the limited results of patient adherence of multivitamin intake after the bariatric surgery. Overall, a poor adherence of multivitamin intake is described, and this topic is described in almost every publication about vitamin deficiencies after the bariatric surgery. However, it remains only at percentages. Only a few studies described a limited number of factors that can affect this adherence. There is insufficient information available, therefore, to perform a systematic review about this subject.

\section{Recommendations for future research}

A prospective cross-sectional study after bariatric surgery is recommended to analyse the different barriers responsible for poor MVS adherence. Beside studying specific patient groups, it is advised to involve various healthcare professionals to educate patients on the nutritional consequences of their obesity treatment. A multidisciplinary approach, facilitating the expertise from all specialties, involved in bariatric care should also include a role for the general practitioner to improve long-term adherence.

\section{Conclusion}

Long-term adherence to MVS after the bariatric surgery is often poor, and there are only limited data on the different factors that influence MVS adherence in bariatric patients. These factors are limited to patient-related factors (age, sex and employment), bariatric surgery-related factors (postoperative complications, gastrointestinal complaints and eating behaviour), therapy-related factors (side effects and composition of MVS), economic factors (costs of MVS) and healthcare-related factors (annually medical visits). A prospective cross-sectional study after the bariatric surgery is recommended to analyse the different barriers responsible for poor MVS adherence. Knowledge gained from studies in other patient populations may therefore be useful for increasing long-term adherence. Patient-centred education is the cornerstone in achieving higher adherence rates, which emphasises the need for dedicated bariatric teams, including dietitians and mental health professionals, and also has an important role for the general practitioner.

\section{Acknowledgments}

Formal ethical approval for this study is not necessary.

H.J.M.S., S.P. and E.J.H. designed the study. H.J.M.S. conducted research and interpretation data. H.J.M.S., S.P., J.F.S. and E.J.H. drafted the article. H.J.M.S., S.P., J.F.S. and E.J. $\mathrm{H}$. were primary responsibility for final content.

All authors read and approved the final manuscript.

\section{References}

1. Angrisani L, Santonicola A, Iovino P, et al. (2018) IFSO Worldwide Survey 2016: primary, endoluminal, and revisional procedures. Obes Surg 28, 3783-3794.

2. Lee GK \& Cha YM (2016) Cardiovascular benefits of bariatric surgery. Trends Cardiovasc Med 26, 280-289.

3. Modi AC, Zeller MH, Xanthakos SA, et al. (2013) Adherence to vitamin supplementation following adolescent bariatric surgery. Obesity 21, E190-E195.

4. Ziegler O, Sirveaux MA, Brunaud L, et al. (2009) Medical follow up after bariatric surgery: nutritional and drug issues. General recommendations for the prevention and treatment of nutritional deficiencies. Diabetes Metab 35, 544-557.

5. Zarshenas N, Tapsell LC, Neale EP, et al. (2020) The relationship between bariatric surgery and diet quality: a systematic review. Obes Surg 30, 1768-1792.

6. Mechanick JI, Apovian C, Brethauer S, et al. (2020) Clinical practice guidelines for the perioperative nutrition, metabolic, and nonsurgical support of patients undergoing bariatric procedures 2019 update: cosponsored by American Association of Clinical Endocrinologists/American College of Endocrinology, the Obesity Society, American Society for Metabolic and Bariatric Surgery, Obesity Medicine Association, and American Society of Anesthesiologists. Obesity 28, O1-O58.

7. Mechanick JI, Youdim A, Jones DB, et al. (2013) Clinical practice guidelines for the perioperative nutritional, metabolic, and nonsurgical support of the bariatric surgery patient-2013 update: cosponsored by American Association of Clinical Endocrinologists, The Obesity Society, and American Society for Metabolic \& Bariatric Surgery. Obesity 21, S1-S27.

8. Aills L, Blankenship J, Buffington C, et al. (2008) ASMBS allied health nutritional guidelines for the surgical weight loss patient. Surg Obes Relat Dis 4, S73-S108.

9. Parrott J, Frank L, Rabena R, et al. (2017) American society for metabolic and bariatric surgery integrated health nutritional guidelines for the surgical weight loss patient 2016 update: micronutrients. Surg Obes Relat Dis 13, 727-741.

10. Ledoux S, Calabrese D, Bogard C, et al. (2014) Long-term evolution of nutritional deficiencies after gastric bypass: an assessment according to compliance to medical care. Ann Surg 259, 1104-1110.

11. Abdeen G \& le Roux CW (2016) Mechanism underlying the weight loss and complications of Roux-en-Y gastric bypass. Rev Obes Surg 26, 410-421.

12. Schijns W, Schuurman LT, Melse-Boonstra A, et al. (2018) Do specialized bariatric multivitamins lower deficiencies after RYGB? Surg Obes Relat Dis 14, 1005-1012.

13. Heusschen L, Schijns W, Ploeger N, et al. (2020) The true story on deficiencies after sleeve gastrectomy: results of a double-blind RCT. Obes Surg 30, 1280-1290.

14. Homan J, Schijns W, Aarts EO, et al. (2016) An optimized multivitamin supplement lowers the number of vitamin and mineral deficiencies three years after Roux-en-Y gastric bypass: a cohort study. Surg Obes Relat Dis 12, 659-667.

15. Smelt HJM, van Loon S, Pouwels S, et al. (2020) Do specialized bariatric multivitamins lower deficiencies after sleeve gastrectomy? Obes Surg 30, 427-438.

16. Ben-Porat T, Elazary R, Goldenshluger A, et al. (2017) Nutritional deficiencies four years after laparoscopic sleeve gastrectomy - are supplements required for a lifetime? Surg Obes Relat Dis 13, 1138-1144.

17. James H, Lorentz P \& Collazo-Clavell ML (2016) Patient-reported adherence to empiric vitamin/mineral supplementation and related nutrient deficiencies after Roux-en-Y Gastric Bypass. Obes Surg 26, 2661-2666.

18. Sunil S, Santiago VA, Gougeon L, et al. (2017) Predictors of vitamin adherence after bariatric surgery. Obes Surg 27, 416-423.

19. Jin J, Sklar GE, Min Sen Oh V, et al. (2008) Factors affecting therapeutic compliance: a review from the patient's perspective. Ther Clin Risk Manage 4, 269-286. 
20. Osterberg L \& Blaschke T (2005) Adherence to medication. N Engl $J$ Med 353, 487-497.

21. Tucker ON, Szomstein S \& Rosenthal RJ (2007) Nutritional consequences of weight-loss surgery. Med Clin North Am 91, 499514, xii.

22. Lizer MH, Papageorgeon H \& Glembot TM (2010) Nutritional and pharmacologic challenges in the bariatric surgery patient. Obes Surg 20, 1654-1659.

23. Malone M (2003) Altered drug disposition in obesity and after bariatric surgery. Nutr Clin Pract 18, 131-135.

24. Smelt HJ, Pouwels S \& Smulders JF (2016) The clinical dilemma of calcium supplementation after bariatric surgery: calcium citrate or calcium carbonate that is the question? Obes Surg 26, 2781-2782.

25. Ahmad DS, Esmadi M \& Hammad H (2012) Malnutrition secondary to non-compliance with vitamin and mineral supplements after gastric bypass surgery: What can we do about it? Am J Case Rep 13, 209-213.

26. Homan J, Schijns W, Janssen IMC, et al. (2019) Adequate multivitamin supplementation after Roux-En-Y gastric bypass results in a decrease of national health care costs: a cost-effectiveness analysis. Obes Surg 29, 1638-1643.

27. Lier HO, Biringer E, Stubhaug B, et al. (2012) The impact of preoperative counseling on postoperative treatment adherence in bariatric surgery patients: a randomized controlled trial. Patient Educ Couns 87, 336-342.

28. Yavuz A, Tuncer M, Erdogan O, et al. (2004) Is there any effect of compliance on clinical parameters of renal transplant recipients? Transplant Proc 36, 120-121.

29. Stone VE, Hogan JW, Schuman P, et al. (2001) Antiretroviral regimen complexity, self-reported adherence, and HIV patients' understanding of their regimens: survey of women in the her study. $J$ Acquir Immune Defic Syndr 28, 124-131.

30. Kaplan RC, Bhalodkar NC, Brown EJ, et al. (2004) Race, ethnicity, and sociocultural characteristics predict noncompliance with lipid-lowering medications. Prev Med 39, 1249-1255.

31. Shaw E, Anderson JG, Maloney M, et al. (1995) Factors associated with noncompliance of patients taking antihypertensive medications. Hosp Pharm 30, 201-203, 6-7.

32. Berghofer G, Schmidl F, Rudas S, et al. (2002) Predictors of treatment discontinuity in outpatient mental health care. Soc Psychiatr Psycbiatr Epidemiol 37, 276-282.

33. Lawson VL, Lyne PA, Harvey JN, et al. (2005) Understanding why people with type 1 diabetes do not attend for specialist advice: a qualitative analysis of the views of people with insulin-dependent diabetes who do not attend diabetes clinic. J Health Psychol 10, 409-423.

34. Farmer KC, Jacobs EW \& Phillips CR (1994) Long-term patient compliance with prescribed regimens of calcium channel blockers. Clin Ther 16, 316-326, discussion 271-272.

35. Claxton AJ, Cramer J \& Pierce C (2001) A systematic review of the associations between dose regimens and medication compliance. Clin Ther 23, 1296-1310.
36. Iskedjian M, Einarson TR, MacKeigan LD, et al. (2002) Relationship between daily dose frequency and adherence to antihypertensive pharmacotherapy: evidence from a meta-analysis. Clin Ther 24, 302-316.

37. De Geest S \& Sabate E (2003) Adherence to long-term therapies: evidence for action. Eur J Cardiovasc Nurs 2, 323.

38. Paes AH, Bakker A \& Soe-Agnie CJ (1997) Impact of dosage frequency on patient compliance. Diabetes Care 20, 1512-1517.

39. Hungin AP, Rubin G \& O'Flanagan H (1999) Factors influencing compliance in long-term proton pump inhibitor therapy in general practice. BrJ Gen Pract 49, 463-464.

40. Seo MA \& Min SK (2005) Development of a structural model explaining medication compliance of persons with schizophrenia. Yonsei Med J 46, 331-340.

41. Gascon JJ, Sanchez-Ortuno M, Llor B, et al. (2004) Why hypertensive patients do not comply with the treatment: results from a qualitative study. Fam Pract 21, 125-130.

42. Christensen DB (1978) Drug-taking compliance: a review and synthesis. Health Serv Res 13, 171-187.

43. Kyngas H \& Rissanen M (2001) Support as a crucial predictor of good compliance of adolescents with a chronic disease. J Clin Nurs 10, 767-774.

44. Lacro JP, Dunn LB, Dolder CR, et al. (2002) Prevalence of and risk factors for medication nonadherence in patients with schizophrenia: a comprehensive review of recent literature. J Clin Psychiatry 63, 892-909.

45. Sewitch MJ, Abrahamowicz M, Barkun A, et al. (2003) Patient nonadherence to medication in inflammatory bowel disease. Am J Gastroenterol 98, 1535-1544.

46. Svarstad BL, Shireman TI \& Sweeney JK (2001) Using drug claims data to assess the relationship of medication adherence with hospitalization and costs. Psychiatr Serv 52, 805-811.

47. Ellis JJ, Erickson SR, Stevenson JG, et al. (2004) Suboptimal statin adherence and discontinuation in primary and secondary prevention populations. J Gen Intern Med 19, 638-645.

48. Spikmans FJ, Brug J, Doven MM, et al. (2003) Why do diabetic patients not attend appointments with their dietitian? J Hum Nutr Diet 16, 151-158.

49. Thakkar J, Kurup R, Laba TL, et al. (2016) Mobile telephone text messaging for medication adherence in chronic disease: a meta-analysis. JAMA Intern Med 176, 340-349.

50. Ramsey RR, Holbein CE, Powers SW, et al. (2018) A pilot investigation of a mobile phone application and progressive reminder system to improve adherence to daily prevention treatment in adolescents and young adults with migraine. Cephalalgia 38, 20352044.

51. Donovan JL \& Blake DR (1992) Patient non-compliance: deviance or reasoned decision-making? Soc Sci Med 34, 507-513.

52. Tichansky DS, Boughter JD \& Madan AK (2006) Taste change after laparoscopic Roux-en-Y gastric bypass and laparoscopic adjustable gastric banding. Surg Obes Relat Dis 2, 440-444.

53. Allen LV (2008) Dosage form design and development. Clin Ther 30, 2102-2111. 